\title{
Aprendizaje de los estudiantes de educación superior a través del itinerario en sitios
} arqueológicos de Lima, Perú

Learning of Higher Education students through the itinerary in archaeological sites of Lima, Perú

Recibido el 28/ 07/ 2020. Revisión del 16/ 08/ 2020. Aceptado 25/09/2020

\author{
Ivan Ernesto Quijano Aranibar
}

Correo: ivan.quijano1@unmsm.edu.pe

Código: https://orcid.org/0000-0003-2264-1186-

Egresado de Maestría en Educación con mención en Docencia Universitaria

Universidad Nacional Mayor de San Marcos

Ofelia Santos Jimenez Carmen

Correo: Código: Ofelia.santos@unmsm.edu.pe

http://orcid.org/0000-0003-1294-0641

Directora de la Unidad de Posgrado de la Facultad de Educación de la UNMSM 


\title{
Resumen
}

El objetivo de estudio fue describir el nivel de aprendizaje de los estudiantes que participaron a través del itinerario en dos yacimientos arqueológicos, uno reconstruido y otro consolidado. El enfoque de investigación es cuantitativo y el tipo descriptivo. Se ha elegido el diseño no experimental de corte transversal. La muestra fue de tipo no probabilística y está constituida por 20 educandos del $3^{\circ}$ ciclo de la carrera de Guía Oficial de Turismo. Se aplicó la encuesta directa a través del instrumento PQ1. De igual manera, se utilizó para el análisis el programa IBM SPSS para obtener las medidas de tendencia. Los resultados demuestran que el estudiantado aprende contenidos conceptuales in situ en los yacimientos arqueológicos por medio del itinerario, pero estos se limitan a la estática y a la dinámica en los niveles 2 y 3 del aprendizaje.

Palabras clave: aprendizaje, educación no formal, educación patrimonial, sitio arqueológico, Perú.

\begin{abstract}
The objective is to describe the level of learning of the students who participated through the itinerary in the reconstructed and consolidated archaeological site. The research approach is quantitative and the type descriptive. The non-experimental cross-sectional design has been chosen. The sample is non-probabilistic and consists of 20 students from the 3rd cycle of the Official Tourism Guide profession. The survey was applied through the PQ1 instrument. In addition, the IBM SPSS program was used for the analysis to obtain the central measures. The results show that students learn concepts in archeological sites through the itinerary, but these are based on static and dynamic levels 2 and 3 of learning.
\end{abstract}

Key words: archaeological sites, heritage education, learning, nonformal education, Perú. 


\section{Introducción}

El patrimonio cultural es toda expresión material e inmaterial reconocida mediante los valores asignados por la sociedad y/o el Estado en un contexto histórico particular (Ballart \& JuanTresserras, 2001), de tal modo que es un constructo sociocultural (Cuenca, 2014). Asimismo, el Ministerio de Cultura (s. f.) ha clasificado el patrimonio peruano a base de su especialización en: material, inmaterial, subacuático, industrial y documental. Sin embargo, de este gran abanico, se ha tratado el patrimonio material de corte arqueológico, definido por las normativas, como el cúmulo de bienes históricos muebles e inmuebles, ubicados en la superficie o en el subsuelo, e investigados por metodologías provenientes de la arqueología (Ibáñez, 2014). En este norte, sólo se enfatiza el valor de uso, sostenido por Ballart (1997), de los elementos patrimoniales arqueológicos inmuebles, ya que cuentan con un conjunto de utilidades para satisfacer las necesidades de índole educativa, turística e inclusive social; por lo que han perdido su uso original, debido a que su función axial ha sido quebrantada (Pérez-Juez, 2006).

A inicios del siglo XXI, el Instituto Nacional de Cultura, hoy Ministerio de Cultura, realizó el primer inventario preliminar de sitios arqueológicos ${ }^{1}$ del Perú y se estima que existen más de 350 yacimientos en Lima Metropolitana (Ramos, 2001), de distinto período y funcionalidad, amparados por la Ley General del Patrimonio Cultural de la Nación N ${ }^{\circ} 28296$ (Instituto Nacional de Cultura, 2007). Por consiguiente, se posee un amplio espectro de inmuebles arqueológicos Precoloniales potenciables desde el punto de vista educativo. No obstante, de este grueso, menos del 3\% están preparados y adaptados formalmente para recibir a la sociedad mediante los modelos de presentación al público. Por un lado, destaca el tipo consolidado, cuya característica radica en congelar el sitio arqueológico en el estado como ha sido dejado luego de la excavación y, por lo tanto, busca conservar los elementos arquitectónicos como testigos del paso del tiempo; y por otro, sobresale el modelo de presentación reconstruido, encargado de completar las partes que faltan del complejo arqueológico por medio de un estudio multidisciplinario y la formulación de hipótesis (Masriera-Esquerra, 2007, 2008; Santacana, 1995; Santacana \& Masriera-Esquerra, 2012).

En el marco de la educación no formal los yacimientos arqueológicos en general poseen las condiciones para ser tratados como recursos didácticos, desde una concepción integral a partir de la cohesión de los medios, materiales y estrategias (Quijano, 2016, 2018), pues permiten construir y fomentar escenarios activos de aprendizaje. Por tanto, nos situamos dentro de la educación patrimonial, entendida como una línea de investigación sobre el proceso de

\footnotetext{
${ }^{1}$ Son utilizados como sinónimos los términos sitio, asentamiento, yacimiento, complejo e inmueble arqueológico, a pesar de su complejidad y particularidad en la literatura netamente arqueológica.
}

Contacto: revistaeduca@umch.edu.pe_ISSN: 2617-0337 revistas.umch.edu.pe/ 
enseñanza-aprendizaje de los fenómenos patrimoniales (Quijano, 2019), dado que utiliza una amplia gama de estrategias, a través de los conjuntos patrimoniales, con el objetivo de comprender la realidad sociocultural e histórica del alumnado (Bardavio \& Mañé, 2017; Cuenca, 2014). Por su parte, el itinerario en el nivel Superior, es el único modelo educativo patrimonial aplicado en los yacimientos arqueológicos de Lima Metropolitana, independientemente si están adaptados o no para su visita e interpretación. Asimismo, a pesar que existe una mayor diversidad de actividades patrimoniales de corte lúdico en la Educación Básica, también tiene este cierta hegemonía. Al respecto, se parte del axioma que los educandos aprenden contenidos en los sitios arqueológicos mediante el itinerario (Valderrama, 2014), realizado por los orientadores, guías de turismo y especialistas en el tema, sea el caso de arqueólogos, historiadores, etc. Inclusive, se concluye, sin evidencia empírica, que las salidas de campo dirigidas a los docentes permiten enriquecer su formación, ya que asimilaron nuevos conocimientos y experiencias en los inmuebles arqueológicos (González, 2012). Sin embargo, la Universidad de Barcelona se ha centrado en evaluar los aprendizajes de los estudiantes a base del tipo de presentación de los complejos arqueológicos. Por ende, concluye que en los sitios reconstruidos existe un aprendizaje real; mientras en los yacimientos consolidados, solo conservados, se evidencia un desaprendizaje (Masriera-Esquerra, 2007, 2008). De este modo, si los estudiantes, a priori, aprenden en los yacimientos arqueológicos gracias al itinerario, cabe preguntarse cuál es el nivel de aprendizaje y, a partir de esto, conocer qué tipos de contenidos conceptuales se están asimilando. Para esta última interrogante, se emplearán los criterios netamente arqueológicos establecidos por Binford (1991) sobre la estática (materialidad arqueológica) y la dinámica (modo de visa) e incluso se sumará el taxón denominado metodología del arqueólogo. En consecuencia, se propone como objetivo general describir el nivel de aprendizaje de los estudiantes que participaron por medio del itinerario en dos yacimientos arqueológicos, uno reconstruido y otro consolidado.

\section{Metodología}

\section{Enfoque, alcance y diseño de investigación}

La investigación es un estudio piloto de enfoque cuantitativo y de alcance descriptivo. Se utilizó el diseño no experimental de corte transversal para especificar el objeto de estudio (Hernández, Fernández, \& Baptista, 2014). En esta línea, se describirá el nivel de aprendizaje de los estudiantes que han participado, por medio del itinerario, en un yacimiento arqueológico reconstruido (YR) y en un yacimiento arqueológico consolidado (YC); por lo que el presente estudio preliminar se enmarca dentro de la educación patrimonial en inmuebles Precoloniales.

\section{Población y muestra}

La población compete a un grupo de 21 educandos del $3^{\circ}$ ciclo, del turno mañana de la carrera de Guía Oficial de Turismo, del Instituto de Educación Superior CEPEA del distrito de Pueblo Libre, Lima, Perú, matriculados en el curso de Arte Prehispánico durante el primer semestre académico de 2019. El alumnado es mixto y su edad oscila entre los 18 y 24 años. Por su parte, la muestra es de tipo no probabilística y ha sido determinada con el programa estadístico Decision Analyst STATS 2.0 y se consideró un margen de error de 5\% y un nivel de confianza al 95\%. Por tanto, la muestra está constituida por 20 alumnos. 


\section{Técnicas e instrumentos}

La técnica empleada ha sido la encuesta. Por ello, se elaboró el cuestionario PQ1 sobre el aprendizaje de contenidos conceptuales sobre los yacimientos arqueológicos Precoloniales, constituido por 20 preguntas cerradas (ítems del 1-20) con varias opciones de respuesta, ver tabla 1. En razón al ítem 8 , del indicador conoce la cultura material, se ha adaptado a la historia particular del YR (8a) y del YC (8b).

\section{Tabla 1}

Ítems del cuestionario $P Q 1$

\begin{tabular}{cl}
\hline $\mathbf{N}^{\circ}$ & \multicolumn{1}{c}{ Ítems } \\
\hline 1 & ¿Qué cultura Prehispánica ha edificado el sitio arqueológico? \\
2 & ¿Qué periodo/etapa arqueológica se ha evidenciado en el yacimiento? \\
3 & ¿En qué valle se ubica el sitio arqueológico? \\
4 & ¿Qué formas y elementos arquitectónicos tiene el yacimiento? \\
5 & ¿Cuál es la técnica constructiva empleada en los muros? \\
6 & ¿Qué materiales se han utilizado en los muros? \\
7 & ¿Qué técnica se utilizó en los rellenos constructivos y cuáles fueron los materiales de \\
& relleno? \\
8 a & ¿Los Lima se enterraban en posición flexionada? \\
$8 b$ & ¿El sitio presenta frisos polícromos con diseños de seres híbridos? \\
9 & ¿Cuál es la función del sitio arqueológico? \\
10 & ¿Cuál es la orientación del sitio arqueológico? \\
11 & ¿El yacimiento arqueológico, a pesar que tuvo una larga duración, ha tenido una tradición \\
& arquitectónica (continuidad) durante su período/etapa de funcionamiento? \\
12 & ¿El sitio arqueológico ha tenido cambios y remodelaciones arquitectónicas durante su \\
& etapa/período de funcionamiento? \\
13 & ¿El sitio arqueológico como es apreciado hoy en día, ha sido construido de manera \\
& secuencial? \\
14 & ¿Cuál es la organización sociopolítica establecida en el sitio arqueológico? \\
15 & ¿Qué actividades económicas se han realizado en el sitio arqueológico? \\
16 & ¿La sociedad asentada en el sitio practicó el trueque para aprovisionarse de recursos no \\
& locales (foráneos)? \\
17 & Observe el plano del yacimiento e identifique sus sectores \\
18 & ¿La élite practicó rituales en el sitio arqueológico a base de la cosmovisión andina? \\
19 & ¿Qué métodos arqueológicos se han utilizado para investigar en el sitio arqueológico? \\
20 & ¿La investigación arqueológica ha sido auxiliada y/o complementada a través de otras \\
& ciencias/disciplinas? \\
\hline
\end{tabular}

Nota: elaboración propia.

Desde luego, para precisar la validez del instrumento, se optó por la modalidad de juicio de expertos. Asimismo, la encuesta se aplicó al finalizar inmediatamente el itinerario, con una duración no mayor a 30 minutos. A partir de esto, la información recolectada ha sido sistematizada con la finalidad de precisar la confiabilidad a través del coeficiente de consistencia interna alfa de Cronbach, ver tabla 2. 


\section{Tabla 2}

Coeficiente de alfa de Cronbach en el instrumento PQ1

\begin{tabular}{ccc}
\hline Sitio/Coeficiente & Alfa de Cronbach & $\mathbf{N}^{\circ}$ de elementos \\
\hline Reconstruido & .563 & 20 \\
\hline Consolidado & .751 & 20 \\
\hline
\end{tabular}

Nota: elaboración propia.

\section{El itinerario en yacimientos arqueológicos}

Los discentes han interactuado en los sitios arqueológicos por medio del itinerario, este consiste en un recorrido por un espacio cultural y natural con paradas durante el trayecto (Coma, 2011). Los encargados de interpretar los inmuebles Prehispánicos han sido los guías oficiales de turismo, mediadores de los yacimientos arqueológicos visitados, tanto para el consolidado como para el reconstruido. En ambos casos, se realizó el recorrido tradicional, ofertado, con una duración no mayor a 90 minutos.

\section{Dimensiones}

El instrumento PQ1 ha sido diseñado a partir de la variable aprendizaje del yacimiento arqueológico. En esta oportunidad, solo se presenta la dimensión conceptual con sus respectivos indicadores. De igual manera, se incluye un taxón arqueológico para su sistematización, ver tabla 3 .

\section{Tabla 3}

Operacionalización de la variable a partir de su dimensión e indicadores

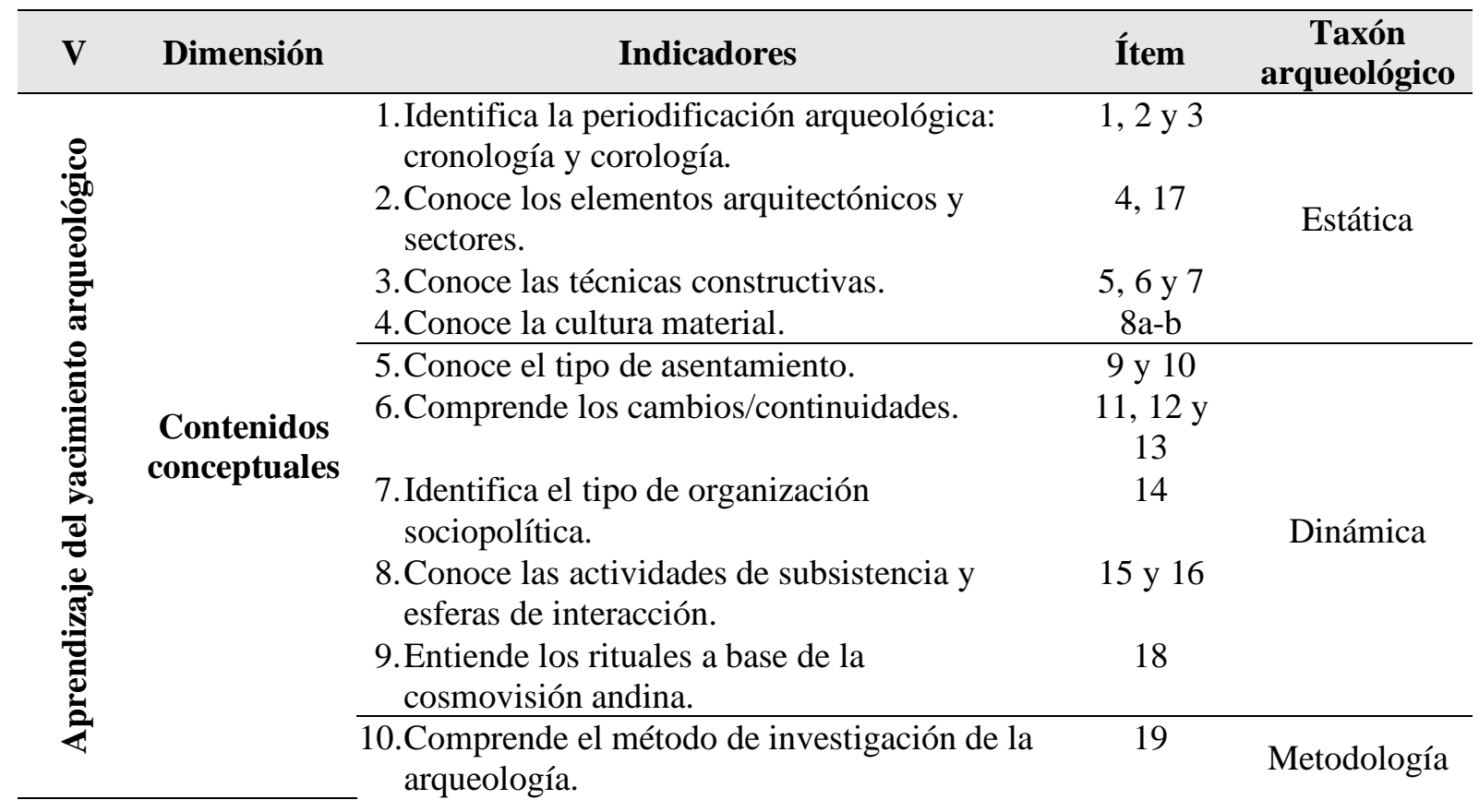

Contacto: revistaeduca@umch.edu.pe_ISSN: 2617-0337 revistas.umch.edu.pe/ 
Nota: abreviación V=Variable. Elaboración propia.

Con respecto a la ponderación de los contenidos conceptuales de la variable aprendizaje del yacimiento arqueológico, se ha establecido un puntaje máximo de 20; por lo que cada ítem equivale a un punto $(20 \times 1=20)$. Asimismo, se han considerado 4 niveles de aprendizaje: nivel 1 de 0 a 5 (N1), nivel 2 de 6 a 10 (N2), nivel 3 de 11 a 15 (N3) y nivel 4 de 16 a 20 (N4).

\section{Resultados, análisis y discusión}

Luego de recolectar y ordenar los datos obtenidos mediante la aplicación del instrumento PQ1, se muestran los resultados de investigación con la finalidad de alcanzar el objetivo general: describir el nivel de aprendizaje de los estudiantes que participaron a través del itinerario en dos sitios arqueológicos, uno reconstruido y otro consolidado. Para ello, se toma en cuenta la dimensión contenidos conceptuales y su respectivo taxón arqueológico, ver tabla 4.

\section{Tabla 4}

Descripción básica de resultados a partir del objetivo general

\footnotetext{
Objetivo general: describir el nivel de aprendizaje de los estudiantes que participaron a través del itinerario en dos sitios arqueológicos, uno reconstruido y otro consolidado.

Ítem Dimensión sobre los contenidos conceptuales sobre la estática

1 El 70\% y $40 \%$ de alumnos que han participado en el YR y en el YC respectivamente, han identificado las culturas Prehispánicas que han construido los complejos arqueológicos; mientras el $30 \%$ y $60 \%$ de estudiantes que visitaron ambos sitios, no han entendido este elemento corológico.

2 El 80\% y $100 \%$ de educandos que han interactuado en el YR y en el YC respectivamente, han identificado los períodos de los yacimientos arqueológicos; a diferencia del $20 \%$ de discentes del primer complejo, que no ha comprendido este elemento cronológico.

3 El 100\% y 90\% de educandos que han participado en el YR y en el YC respectivamente, han identificado en qué valle se ubican los mencionados complejos; en contraste del $10 \%$ de alumnos, que no ha logrado identificar en el YC este elemento de la corología.

4 El 10\% y $50 \%$ de educandos que han interactuado en el YR y en el YC respectivamente, han conocido los elementos arquitectónicos de los complejos arqueológicos; en oposición al 90\% y $50 \%$ de estudiantes, que no han entendido estos criterios.

5 El 90\% y $60 \%$ de alumnos que han interactuado en el YR y en el YC respectivamente, han conocido las técnicas constructivas empleadas en los muros; en contraste del $10 \%$ y $40 \%$ de educandos que no han logrado identificar estos elementos de la arquitectura en ambos yacimientos.

6 El 100\% y 60\% de estudiantes que han participado en el YR y en el YC, han comprendido los materiales utilizados en los muros; en oposición al $40 \%$ de discentes, que no ha conocido estos insumos en los complejos consolidados.

7 El 70\% y 80\% de educandos que han interactuado en el YR y en el YC respectivamente, han conocido las técnicas utilizadas en los rellenos constructivos y sus materiales de relleno; a
}

Contacto: revistaeduca@umch.edu.pe_ISSN: 2617-0337 revistas.umch.edu.pe/ 
diferencia del $20 \%$ y $30 \%$ de educandos, que no han comprendido estas técnicas empleadas en los cimientos de ambos sitios.

8 El 70\% y 90\% de alumnos que han participado en el YR y en el YC respectivamente, han conocido la cultura material; en contraste del $30 \%$ y $10 \%$ de estudiantes que no han entendido la materialidad arqueológica en ambos sitios

\begin{tabular}{cc}
\hline Ítem & \multicolumn{1}{c}{ Dimensión sobre los contenidos conceptuales sobre la dinámica } \\
\hline 9 & $\begin{array}{l}\text { El 30\% y 70\% de educandos que han interactuado en el YR y en el YC respectivamente, han } \\
\text { conocido la funcionalidad de los complejos arqueológicos; en oposición al } 70 \% \text { y 30\% de } \\
\text { discentes, que no han comprendido este rasgo de los asentamientos visitados. }\end{array}$
\end{tabular}

10 El $100 \%$ y $50 \%$ de estudiantes que han visitado el YR y el YC respectivamente, han entendido la orientación de los complejos arqueológicos; a diferencia del 50\% de educandos, que no ha logrado conocer este rasgo en los YC.

11 El 70\% y 90\% de discentes que han visitado el YR y el YC respectivamente, han comprendido la tradición arquitectónica de los complejos arqueológicos; a diferencia del 30\% y 10\% de educandos, que no han entendido esta dimensión diacrónica de los yacimientos.

12 El 90\% y 70\% de educandos que han participado en el YR y en el YC respectivamente, han comprendido los cambios y remodelaciones arquitectónicas de los yacimientos; en oposición al $10 \%$ y $30 \%$ de alumnos, que no han entendido esta dinámica en los sitios arqueológicos.

13 El 90\% y $80 \%$ de discentes que han interactuado en el YR y en el YC, han comprendido que ambos sitios han sido construidos de manera secuencial; a diferencia del $10 \%$ y $20 \%$ de educandos, que no han entendido los cambios/continuidades de los complejos arqueológicos.

14 El 10\% y $60 \%$ de alumnos que han participado en el YR y en el YC respectivamente, han identificado la organización sociopolítica establecida en los complejos; en contraste del $90 \%$ y $40 \%$ de educandos que no han reconocido estas categorías en los yacimientos arqueológicos.

15 El 30\% de estudiantes que ha visitado el YR, al igual que el 30\% de discentes que ha participado en el YC, han conocido la actividad de subsistencia practicada en los complejos mencionados; mientras el $70 \%$ de educandos, que ha interactuado en ambos sitios arqueológicos, no ha comprendido estas prácticas económicas.

16 El 30\% de alumnos que ha participado en el YR, al igual que el 30\% de estudiantes que ha interactuado en el YC, han conocido el trueque como mecanismo de captación de bienes no locales; a diferencia del $70 \%$ de educandos, que no ha comprendido esta práctica económica en ambos complejos arqueológicos.

17 El 20\% y 70\% de los discentes que han visitado el YR y el YC respectivamente, han observado los planos e identificado los sectores de los yacimientos arqueológicos; a diferencia del $80 \%$ y $30 \%$ de educandos quienes no han identificado estos sectores en ambos sitios.

18 El 40\% de discentes que ha interactuado en el YR, al igual que el 40\% de educandos que ha participado en el YC, han entendido los principales rituales realizados en los yacimientos aludidos a base de la cosmovisión andina; en contraste del 60\% de alumnos que no ha comprendido estas actividades ceremoniales en ambos complejos.

\begin{tabular}{cl}
\hline Ítem & Dimensión sobre los contenidos conceptuales sobre la metodología de la arqueología \\
\hline 19 & $\begin{array}{l}\text { El } 40 \% \text { y } 10 \% \text { de educandos que han interactuado en el YR y en el YC respectivamente, han } \\
\text { comprendido los métodos arqueológicos usados para investigar los complejos aludidos; en } \\
\text { oposición al } 60 \% \text { y } 90 \% \text { de discentes, que no han entendido el tratamiento de los }\end{array}$ \\
$\begin{array}{l}\text { asentamientos arqueológicos. } \\
\text { El } 20 \% \text { y } 70 \% \text { de estudiantes que han visitado el YR y el YC respectivamente, han entendido } \\
\text { que la investigación de los complejos arqueológicos ha sido auxiliada y/o complementada, a } \\
\text { través de otras ciencias/disciplinas; a diferencia del } 80 \% \text { y 30\% de discentes, que no han } \\
\text { entendido este carácter multidisciplinario de la arqueología. }\end{array}$ \\
\hline
\end{tabular}

Nota: abreviaciones $\mathrm{YR}=$ Yacimiento Reconstruido/ $\mathrm{YC}=$ Yacimiento Consolidado. Elaboración propia. 
Seguidamente, respecto al análisis de datos, se utilizó el programa IBM SPSS versión 20.0 para obtener las medidas de tendencia. Desde luego, con una escala del 0 al 20, el estudiantado que participó en el YR obtuvo una media de 11.1 en la ponderación final; mientras en el YC, alcanzó una puntuación de 11.9. Por ende, probablemente, las medias no evidencian diferencias significativas y manifiestan que el grueso de los discentes no alcanzó los promedios esperados, es decir una ponderación entre 16 y 20 (N4). Asimismo, el alumnado que interactuó en el primer yacimiento mediante el itinerario, tiene una moda de 12 puntos; mientras en el segundo sitio, obtuvo una de 10. Entonces, son las puntuaciones de mayor frecuencia al finalizar esta actividad educativa patrimonial y, al parecer, no se muestran diferencias significativas entre ellas, pues solamente se diferencian en 2 puntos y, por tanto, no se ubican en los límites deseados (N4). Encima, en el YR y en el YC, los educandos alcanzaron una mediana de 11.5 y de 10.5 respectivamente; por lo que se expresa que la mitad de ellos han asimilado contenidos conceptuales por debajo y por arriba de estas puntuaciones. A pesar de esto, la mayoría de los discentes no han logrado alcanzar el intervalo esperado (N4), teniendo en cuenta que los valores máximos y mínimos son de 7 y 16 para el primer sitio y de 8 y 19 para el segundo complejo e, inclusive, considerando que el 10\% de ellos ha obtenido un puntaje de 16 en el YR y, de igual forma, sólo el $10 \%$ ha alcanzado el puntaje de 19 en el YC, ver tabla 5.

\section{Tabla 5}

Distribución de frecuencias en los sitios arqueológicos

\begin{tabular}{cccccc}
\hline \multicolumn{4}{c}{ Yacimiento arqueológico } \\
\hline \multicolumn{3}{c}{ Reconstruido } & \multicolumn{3}{c}{ Consolidado } \\
\hline $\mathbf{X}_{\mathbf{i}}$ & $\mathbf{f}$ & $\%$ & $\mathbf{X}_{\mathbf{i}}$ & $\mathbf{f}$ & $\%$ \\
7 & 1 & $10 \%$ & 8 & 1 & $10 \%$ \\
8 & 1 & $10 \%$ & 9 & 1 & $10 \%$ \\
9 & 1 & $10 \%$ & 10 & 3 & $30 \%$ \\
11 & 2 & $20 \%$ & 11 & 1 & $10 \%$ \\
12 & 3 & $30 \%$ & 13 & 1 & $10 \%$ \\
13 & 1 & $10 \%$ & 14 & 1 & $10 \%$ \\
16 & 1 & $10 \%$ & 15 & 1 & $10 \%$ \\
& & & 19 & 1 & $10 \%$ \\
\hline Total & 10 & $100 \%$ & Total & 10 & $100 \%$ \\
\hline
\end{tabular}

Nota: elaboración propia

En suma, en función al aprendizaje de los estudiantes que participaron gracias al itinerario en ambos sitios arqueológicos (YR y YC), se precisa que solo el 50\% se halla en el N3, es decir con una ponderación entre 11 y 15; a diferencia del $40 \%$ que se sitúa en el N2, a saber, entre 6 y 10 puntos; y solo el 10\%, se circunscribe en el N4, entre 16 y 20 , ver figura 1.

Contacto: revistaeduca@umch.edu.pe_ISSN: 2617-0337 revistas.umch.edu.pe/ 


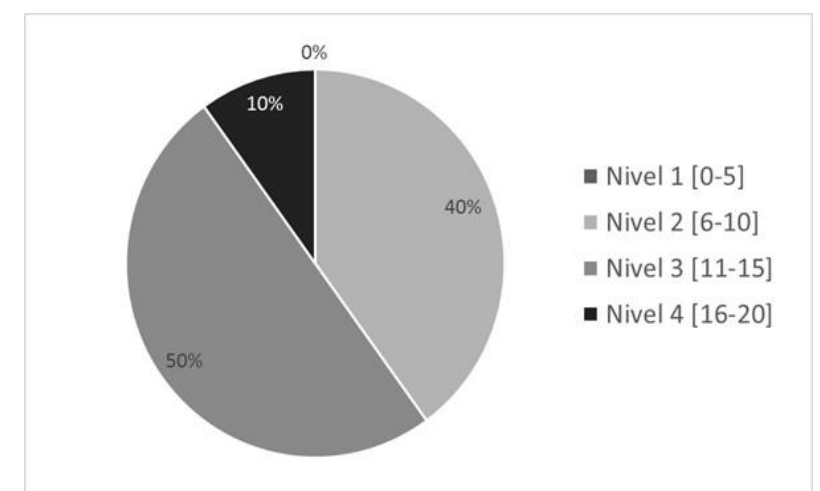

Figura 1. Niveles de aprendizaje de los estudiantes que han participado gracias al itinerario en los sitios arqueológicos. Elaboración propia.

En este escenario, el aprendizaje de los estudiantes en el YR, se limita a un $28 \%$ de contenidos conceptuales vinculados a la estática y un $24.5 \%$ sobre la dinámica; a diferencia de un $3 \%$ de contenidos que se focalizan en la metodología de la arqueología, ver figura 2.

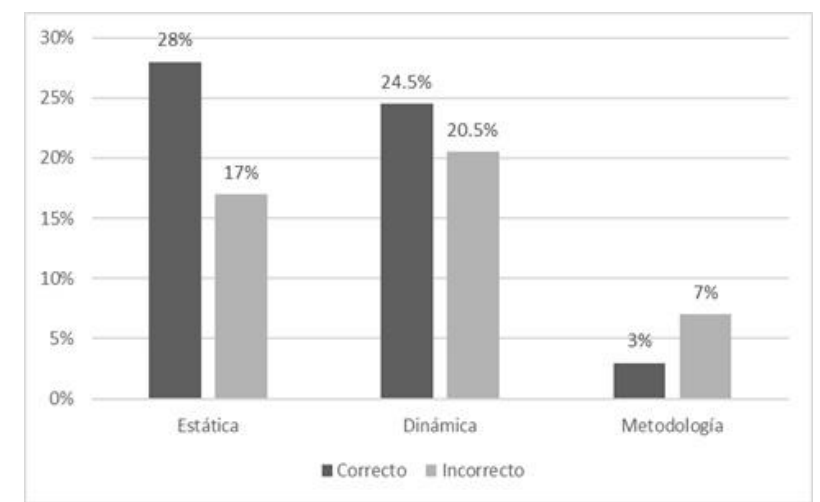

Figura 2. Aprendizaje en el yacimiento reconstruido a base del taxón arqueológico. Elaboración propia.

Además, un panorama semejante se expresa en el YC, debido a que el $29.5 \%$ y $26 \%$ asimiló contenidos referidos al primer y segundo taxón arqueológico e igualmente un menor porcentaje se limita a la metodología de la arqueología (4\%), ver figura 3. 


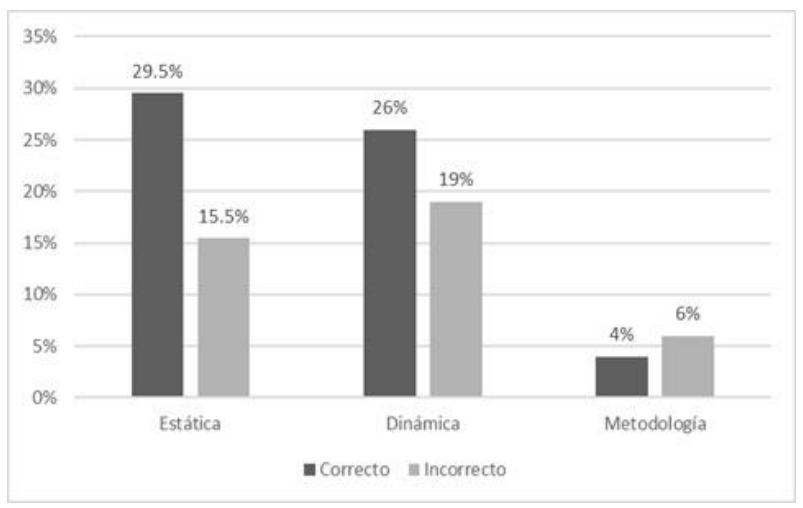

Figura 3. Aprendizaje en el yacimiento consolidado a base del taxón arqueológico. Elaboración propia.

En cuanto a la discusión, los resultados son gratificantes, dado que son similares a los establecidos, literalmente, por Valderrama (2014) y González (2012), debido a que el estudiantado aprende contenidos conceptuales in situ en los sitios arqueológicos mediante el itinerario. Sin embargo, se pone de manifiesto, con evidencia empírica, que el nivel de asimilación de los discentes no es el esperado, pues el $40 \%$ se sitúa en el N2 (6 y 10 puntos) y solo el 50\% se localiza en el N3 (11 y 15 puntos). Asimismo, los aprendizajes han sido limitados, tanto en el YR como en el YC; a diferencia de los resultados de Masriera-Esquerra (2007, 2008), quien ha sostenido que solo el alumnado aprende en los YR y, por su parte, en los YC desaprende. De igual forma, los resultados colectados, a partir de la construcción y aplicación del instrumento PQ1, y clasificados a base de la propuesta de Binford (1991) y del taxón metodología, han sido de gran utilidad, porque se han sistematizado los tipos de contenidos conceptuales asimilados sobre los yacimientos arqueológicos.

\section{Conclusiones}

A base de la evidencia empírica, se concluye, de manera preliminar, que el estudiantado del $3^{\circ}$ ciclo de la carrera de Guía Oficial de Turismo, que participo in situ, a través del itinerario, en un yacimiento arqueológico reconstruido (YR) y en un sitio arqueológico consolidado (YC), aprende contenidos conceptuales sobre ambos complejos, pero el nivel de asimilación es limitado y se circunscribe principalmente a los niveles 2 y 3 . Además, estos contenidos se focalizan en la dinámica y la estática; mientras los referentes a la metodología de la arqueología son prácticamente nulos por lo que los educandos, no entienden el proceso de investigación del arqueólogo (método científico). Al respecto, en relación a la estática, se asimiló la periodificación arqueológica (cronología/corología) y la cultura material; entre tanto, sobre la dinámica, se comprendió principalmente los cambios y continuidades en ambos sitios. Por su parte, al parecer, no hay diferencias significativas entre el aprendizaje de contenidos conceptuales en el YR y en el YC. En suma, el presente trabajo de corte descriptivo, más que conclusiones finales, nos ofrece un panorama general sobre la educación patrimonial en inmuebles Precoloniales, a partir del modelo educativo denominado itinerario. Por ello, servirá de base a futuras investigaciones para ampliar y contrastar nuestros resultados. Asimismo, se desprenden un conjunto de interrogantes a ser tratadas sobre esta línea: ¿Existen diferencias significativas en los aprendizajes de contenidos, por medio del itinerario, en el YR y en el YC?

Contacto: $\underline{\text { revistaeduca@umch.edu.pe_ISSN: 2617-0337_revistas.umch.edu.pe/ }}$ 
¿Por qué se asimilan mediante el itinerario contenidos conceptuales en los niveles 2 y 3 ? ¿Por qué no se aprenden contenidos conceptuales sobre la metodología del arqueólogo (método científico)? ¿Existen diferencias significativas en el aprendizaje del alumnado a través del itinerario realizado por los guías oficiales de turismo y por los arqueólogos e historiadores? ¿Los contenidos conceptuales tratados in situ en los yacimientos arqueológicos, gracias al itinerario, pueden ser asimilados en mayor proporción por otras estrategias y recursos empleados dentro del aula? ¿Qué contenidos procedimentales y actitudinales se asimilan, a partir del itinerario, y en qué medida?

\section{Referencias}

Ballart, J. (1997). El Patrimonio histórico y arqueológico: valor y uso. Barcelona: Editorial Ariel S.A.

Ballart, J., \& Juan-Tresserras, J. (2001). Gestión del patrimonio cultural. Barcelona: Editorial Ariel S.A.

Bardavio, A., \& Mañé, S. (2017). La arqueología en la enseñanza obligatoria. El ejemplo del campo de aprendizaje de la Noguera. Revista Otarq, 2, 331-345. Recuperado de http://revistas.jasarqueologia.es/index.php/otarq/article/view/127/128

Binford, L. R. (1991). En busca del pasado: descifrando el registro arqueológico (2a ed.). Barcelona: Editorial Crítica S.A.

Coma, L. (2011). Actividades educativas y didáctica del patrimonio en las ciudades españolas. Análisis, estado de la cuestión y valoración para una propuesta de modelización (Tesis doctoral, Universitat de Barcelona, Barcelona, España). Recuperado de http://www.tesisenred.net/handle/10803/52205

Cuenca, J. M. (2014). El papel del patrimonio en los centros educativos: hacia la socialización patrimonial. Tejuelo, (19), 76-96. Recuperado de http://rabida.uhu.es/dspace/bitstream/handle/10272/7927/El_papel_del_patrimonio.pdf?s equence $=2$

González, C. (2012). El rol educativo de la arqueología y la didáctica del patrimonio arqueológico. Experiencias y propuestas. Arqueología y Sociedad, (25), 415-435.

Hernández, R., Fernández, C., \& Baptista, P. (2014). Metodología de la investigación (6a ed.). México D.F.: McGraw-Hill.

Ibáñez, M. (2014). Percepción y usos del patrimonio arqueológico de Sevilla. Sevilla: Universidad de Sevilla.

Instituto Nacional de Cultura. (2007). Ley General del Patrimonio Cultural de la Nación 28296 y su reglamento. Recuperado de http://www2.congreso.gob.pe/sicr/cendocbib/con2_uibd.nsf/562A9CCF932F0F6205257 7E300711E65/\$FILE/2Ley_28296.pdf

Masriera-Esquerra, C. (2007). Anàlisi dels espais de presentació arqueològics de l'edat dels metalls (Tesis doctoral, Universitat de Barcelona, Barcelona, España). Recuperado de http://diposit.ub.edu/dspace/handle/2445/41453

Contacto: revistaeduca@umch.edu.pe_ISSN: 2617-0337 revistas.umch.edu.pe/ 
Masriera-Esquerra, C. (2008). Presentación del patrimonio arqueológico: ruinas «versus» reconstrucciones. ¿Qué entiende más el público? Íber. Didáctica de las Ciencias Sociales, Geografía e Historia, (57), 39-51.

Ministerio de Cultura. (s. f.). ¿Qué es patrimonio cultural? Recuperado de https://www.cultura.gob.pe/sites/default/files/paginternas/tablaarchivos/04/1manualquee spatrimonio.pdf

Pérez-Juez, A. (2006). Gestión del patrimonio arqueológico. El yacimiento como recurso turístico. Barcelona: Editorial Ariel S.A.

Quijano, I. (2016). Importancia de la arqueología experimental como recurso didáctico en el proceso de aprendizaje de los estudiantes del curso de Historia del Perú Precolombino (Tesis de licenciatura). Recuperado de http://repositorio.unfv.edu.pe/handle/UNFV/1497

Quijano, I. (2018). El uso de la arqueología experimental como recurso didáctico en el proceso de aprendizaje: Una experiencia educativa en estudiantes de administración turística de Lima, Perú. Revista Electrónica Educare, 22(3). https://doi.org/10.15359/ree.22-3.14

Quijano, I. (2019). Arqueología experimental y competencias pedagógicas investigativas. Una propuesta desde la educación patrimonial en el Perú. Recuperado de https://dialnet.unirioja.es/servlet/libro?codigo=732585

Ramos, J. (2001). Contribución para un primer inventario general de sitios arqueológicos del Perú. Recuperado de http://repositorio.cultura.gob.pe/handle/CULTURA/85

Santacana, J. (1995). Los parques arqueológicos en Europa. Noticia de unos espacios didácticos desconocidos hasta ahora en España. Íber. Didáctica de las Ciencias Sociales, Geografía e Historia, (3), 100-112.

Santacana, J., \& Masriera-Esquerra, C. (2012). La arqueología reconstructiva y el factor didáctico. Gijón: Trea S.L.

Valderrama, Á. (2014). Sistema de talleres de arqueología en la educación básica regular. Arqueología y Sociedad, (24), 237-258.

\section{Datos del autor}

Egresado de la maestría en Educación con mención en Docencia Universitaria por la Universidad Nacional Mayor de San Marcos (UNMSM). Licenciado en Educación por la Universidad Nacional Federico Villarreal (UNFV) y Licenciado en Arqueología por la UNMSM. Sus líneas de investigación tratan sobre la educación patrimonial, didáctica del patrimonio en yacimientos arqueológicos y museos pedagógicos. Ha publicado artículos científicos en revistas Latinoamericanas indexadas en Scopus y SciELO, interrelacionado el campo educativo y arqueológico. Además, ha publicado libros sobre la arqueología experimental como recurso didáctico y las competencias pedagógicas investigativas. Actualmente, es docente e investigador en la Escuela Nacional de Marina Mercante y en otros institutos de Educación Superior. 
Educa UMCH. Revista sobre Educación y Sociedad, 2020, 15(1), 5-22.

https://doi.org/10.15366/Educa UMCH2019.17.3.001 